\section{Avaliação da cascata de cuidado na prevenção da transmissão vertical do HIV no Brasil}

\author{
Evaluation of the cascade of care in prevention \\ of mother-to-child HIV transmission in Brazil
}

\author{
Angelica Espinosa Miranda 1 \\ Gerson Fernando Mendes Pereira 2 \\ Maria Alix Leite Araujo 3 \\ Mariangela Freitas da Silveira 4 \\ Leonor De Lannoy Tavares 5 \\ Leila Cristina Ferreira da Silva 6 \\ Sandra Fagundes Moreira-Silva 7 \\ Valéria Saraceni 8
}

\title{
Resumo
}

Este estudo teve por objetivo avaliar a cascata de cuidado da redução da transmissão vertical do HIV nos estados do Amazonas, Ceará, Espírito Santo, Rio de Janeiro, Rio Grande do Sul e no Distrito Federal, usando dados do Sistema de Informação de Agravos de Notificação (SINAN). Entre os anos de 2007 e 2012, cresceu a taxa de detecção de HIV na gestação em 5 estados, variando de 7,3\% no Distrito Federal a 46,1\% no Amazonas, com redução de 18,6\% no Rio de Janeiro. Menos de 90\% das mulheres usaram antirretroviral durante o pré-natal, incluídas as que já se sabiam portadoras do HIV. A realização de cesárea eletiva foi baixa. A taxa de detecção de AIDS em crianças menores de 5 anos como proxy da transmissão vertical do HIV apresentou uma redução de 6,3\% entre 2007 e 2012, sendo a maior no Rio Grande do Sul (50\%), que apresentou as maiores taxas do período, enquanto no Espírito Santo ocorreu o maior aumento (50\%). A avaliação da cascata do cuidado do HIV na gestante apontou falhas em todos os pontos. $E$ necessária uma conexão entre a atenção básica e os centros de referência para HIVIAIDS, ordenando o cuidado da família e o melhor desfecho para a criança.

HIV; Gravidez; Transmissão Vertical de Doença Infecciosa; Atenção Primária à Saúde

\footnotetext{
1 Universidade Federal do Espírito Santo, Vitória, Brasil. 2 Departamento Nacional de DST, AIDS e Hepatites Virais, Secretaria de Vigilância em Saúde, Ministério da Saúde, Brasília, Brasil.

3 Programa de Pós-graduação em Saúde Coletiva,

Universidade de Fortaleza, Fortaleza, Brasil.

${ }^{4}$ Departamento MaternoInfantil, Universidade Federal de Pelotas, Pelotas, Brasil.

5 Secretaria de Saúde do Distrito Federal, Brasília Brasil.

6 Fundação de Vigilância em Saúde, Secretaria de Estado de Saúde do Amazonas, Manus, Brasil.

7 Programa Estadual de DST/ AIDS, Secretaria de Estado da Saúde do Espírito Santo, Vitória, Brasil.

${ }^{8}$ Secretaria Municipal de Saúde do Rio de Janeiro, Rio de Janeiro, Brasil.
} 


\section{Introdução}

Os padrões de disseminação da infecção pelo vírus da imunodeficiência adquirida (HIV) mudaram, inclusive no Brasil, devido ao predomínio da forma de transmissão heterossexual 1. A tendência de crescimento relativo da epidemia de AIDS em mulheres 2,3, especialmente daquelas em idade reprodutiva, trouxe como consequência a elevação do número de crianças infectadas pela transmissão vertical, que consiste na passagem do vírus da mãe para filho, o que pode ocorrer durante a gestação, o parto ou a lactância 4,5.

A transmissão vertical tem sido responsável pelos casos de AIDS em crianças em todo o mundo 6 e, no Brasil, cerca de $84 \%$ desses casos em crianças com até 13 anos de idade são decorrentes dessa forma de transmissão 1 . A probabilidade de ocorrer a transmissão vertical pode chegar a $25,5 \%$ sem qualquer intervenção. No entanto, por meio de intervenções preventivas, a transmissão pode reduzir-se para níveis entre zero e 2\% 5,7. O relatório da Fundo das Nações Unidas para a Infância (UNICEF) sobre transmissão vertical do HIV apontou uma redução de cerca de $50 \%$ no número de casos novos de AIDS em crianças, no período entre 2001 e 20128.

Um estudo, realizado em uma amostra representativa de parturientes brasileiras entre 15 e 49 anos de idade, revelou que a prevalência de mulheres portadoras do HIV no momento do parto foi de $0,42 \%$, correspondendo a 13 mil infectadas dentre cerca de 3 milhões de parturientes ${ }^{9}$. Em novo estudo nacional, com mais de 23 mil puérperas entre 2011 e 2012, a prevalência de HIV encontrada foi de $0,4 \% 10$, muito semelhante à anterior.

O Ministério da Saúde recomenda como estratégias para a redução da transmissão vertical do HIV o uso de medicamentos antirretrovirais combinados (terapia antirretroviral - TARV) na grávida e no recém-nascido, o parto cesáreo e a não amamentação ${ }^{5}$. Ações encadeadas, desde o acesso ao atendimento pré-natal e à testagem anti-HIV, até a finalização do seguimento da criança exposta, irão garantir o sucesso do programa para a redução da transmissão vertical do HIV 5,11. Entretanto, relato de oportunidades perdidas para essa prevenção continuam a ser publicados no Brasil, como a alta prevalência de mulheres grávidas não testadas 12 , e o baixo acesso ao pacote de medidas preventivas para HIV 13 , resultando, por exemplo, em uma taxa de transmissão vertical do HIV de 9,9\% no Amazonas no período de 2007 a 200914 .

O objetivo deste estudo foi identificar, mediante dados de notificação, as oportunidades perdidas de redução da transmissão vertical do
HIV em cinco estados da federação e no Distrito Federal. O conceito de cascata de cuidado ou continuum of care foi utilizado para acessar os passos que fariam parte desse cuidado e, quando não alcançados, representariam as oportunidades perdidas 11 .

\section{Métodos}

Estudo descritivo dos casos de HIV na gestação, incluindo avaliação ecológica e transversal, conduzido nos estados do Amazonas, Ceará, Distrito Federal, Espírito Santo, Rio de Janeiro e Rio Grande do Sul, seis estados da Federação com representatividade macrorregional dos casos de infecção pelo HIV no período de 2007-2012. Os casos foram aceitos como tendo sido notificados de acordo com a definição de caso de HIV em gestante do Ministério da Saúde. As distribuições proporcionais das variáveis sociodemográficas e clínicas disponíveis no Sistema de Informação de Agravos de Notificação (SINAN) foram comparadas pelo teste de qui-quadrado de Pearson. As medianas de idade foram comparadas por Anova. Os dados foram analisados no pacote estatístico Stata 11.2 e a significância estabelecida ao nível de $5 \%$.

As fontes de informação dos casos de HIV/ AIDS em crianças menores de 13 anos foram o SINAN, o Sistema de Informação sobre Mortalidade (SIM), o Sistema de Controle de Exames Laboratoriais (SISCEL) e o Sistema de Controle Logístico de Medicamentos (SICLOM) da Secretaria de Vigilância em Saúde do Ministério da Saúde.

Como a base de dados sobre crianças expostas ao HIV não se encontra disponível, não foi possível calcular a taxa de transmissão vertical do HIV por unidade federada. Para substituir tal informação, os dados referentes ao indicador de taxa de detecção de AIDS em crianças menores de 5 anos foram retirados do Boletim Epidemiológico do Departamento Nacional de DST/AIDS e Hepatites Virais 1.

O projeto foi aprovado no Comitê de Ética em Pesquisa da Universidade Federal do Espírito Santo, sob o número 640.580/2014.

\section{Resultados}

A taxa de detecção de HIV na gestação por mil nascidos vivos apresentou um incremento em quatro estados e no Distrito Federal entre 2007 e 2012, variando de 7,3\% no Distrito Federal a $46,1 \%$ no Amazonas, com uma redução no Rio de Janeiro de 18,6\%. 
$\mathrm{Na}$ base de dados de gestantes notificadas com HIV, o percentual de municípios "silenciosos”, ou seja, aqueles que não apresentaram nenhuma notificação de HIV na gestação no período foram de 45,2\% no Amazonas, 44\% no Ceará, $35,9 \%$ no Espírito Santo, 16,3\% no Rio de Janeiro e $47,7 \%$ no Rio Grande do Sul, sendo que o último apresenta 497 municípios.

A Tabela 1 apresenta a distribuição proporcional das características sociodemográficas e clínicas das mulheres notificadas com HIV na gestação em seis estados brasileiros, de 2007 2012. A mediana de idade foi de 23 anos no Amazonas, 26 anos no Ceará e Rio de Janeiro e 27 anos no Distrito Federal, Espírito Santo e Rio Grande do Sul. O Amazonas e o Distrito Federal apresentaram maior escolaridade. A heterogeneidade na distribuição de raça/cor acompanhou as características regionais, com maior percentual de indígenas no Amazonas. Esse estado, junto com o Rio de Janeiro, teve uma maior proporção de casos notificados em residentes na capital, enquanto o Ceará, o Espírito Santo e o Rio Grande do Sul distribuíram mais a residência entre outros municípios. A maior parte das gestantes já sabia ser portadora do HIV no Distrito Federal, Espírito Santo e Rio Grande do Sul, mas as residentes no Amazonas, Ceará e Rio de Janeiro descobriram ser portadoras do HIV em maior proporção durante o pré-natal. Ainda assim, no Amazonas, $19,6 \%$ só souberam de sua soropositividade no parto. A realização de pré-natal variou de $79 \%$ no Amazonas a 91\% no Espírito Santo. A utilização de TARV durante o pré-natal foi de $41,6 \%$ no Amazonas e 77,2\% no Espírito Santo, enquanto no parto a variação foi de $37,9 \%$ no Amazonas a $74,2 \%$ no Rio Grande do Sul. A cesárea eletiva foi a via de parto de $32,3 \%$ dos nascimentos no Amazonas e $61,4 \%$ no Distrito Federal. O produto do parto foi de nascidos vivos em sua maioria, mas houve um percentual alto de informação ignorada para a variável no Amazonas $(45,3 \%)$ e Rio de Janeiro (30,8\%). O uso de antirretroviral pelo recém-nascido vivo foi iniciado nas primeiras 24 horas em 87,9\% no Distrito Federal e 95,7\% no Rio Grande do Sul.

Na Tabela 2, encontram-se as distribuições proporcionais, com seus respectivos intervalos de 95\% de confiança (IC95\%), das oportunidades de redução da transmissão vertical do HIV a partir do momento do diagnóstico de HIV das gestantes. Os valores se referem à cascata de ações desenvolvidas para mulheres que foram diagnosticadas com HIV antes da gestação, durante o pré-natal ou na admissão para o parto. Em cada cenário, a gestante precisava estar no grupo selecionado anteriormente para estar no seguinte, isto é, saber ser portadora do HIV, ter feito pré- natal, ter recebido TARV na gestação, ter recebido TARV no parto, ter sido submetida à cesárea eletiva, de modo que a cascata de cuidado fosse avaliada em todos os seus componentes, sendo sempre considerados os produtos vivos das gestações para o tempo de início de antirretroviral no recém-nascido.

Para as gestantes que já conheciam o status sorológico de HIV antes da gestação que deu origem à notificação a proporção de realização de pré-natal foi mais baixa no Amazonas (81,7\%) e mais alta no Espírito Santo (94,2\%). A utilização de TARV durante a gestação foi menor no Amazonas $(68,3 \%)$ e maior no Distrito Federal $(89,9 \%)$ e Espírito Santo (89,8\%). A utilização de TARV no momento do parto correspondeu a $24,1 \%$ no Amazonas e $64,9 \%$ no Distrito Federal. A via de parto foi a cesárea eletiva em $18,7 \%$ das mulheres do Amazonas e 53,6\% naquelas do Ceará. Entre os recém-nascidos vivos, a TARV foi iniciada nas primeiras 24 horas de vida em 18\% no Amazonas e 51,9\% no Ceará.

Quando o diagnóstico de HIV foi realizado durante o pré-natal, a utilização de TARV durante a gravidez variou de 50,8\% no Amazonas a 82,6\% no Espírito Santo, e a utilização de TARV no parto foi novamente menor no Amazonas $(20,9 \%)$ e maior no Distrito Federal (61,8\%), proporcionalmente. A cesárea eletiva como via de parto ocorreu em 16,7\% das parturientes do Amazonas e $54,2 \%$ daquelas no Distrito Federal. A TARV para os recém-nascidos vivos iniciou-se nas primeiras 24 horas em 16,5\% no Amazonas e 50,4\% no Distrito Federal.

No caso das gestantes com diagnóstico somente na hora do parto, verificou-se que entre $45,8 \%$ (Rio de Janeiro) e 65,7\% (Ceará) delas informaram ter comparecido ao atendimento pré-natal sem, entretanto, referir testagem para o HIV. Ainda foi possível utilizar TARV no parto em $60 \%$ daquelas do Distrito Federal e 75,5\% no Ceará. Os recém-nascidos vivos receberam TARV nas primeiras 24 horas em 43,5\% das vezes no Rio de Janeiro e em 70,6\% no Ceará.

Os 1.780 casos incluídos na análise de casos de AIDS em crianças menores de 13 anos de idade e representando os 5 estados e o Distrito Federal tiveram suas informações extraídas do SISCEL em 1.681 casos, 448 do SINAN e 122 do SIM. A informação encontrava-se disponível apenas no SISCEL em 1246 casos, apenas no SINAN em $48 \mathrm{e}$ foram retirados do SIM somente 41 casos. Vinte e seis casos estavam nos 3 sistemas, 364 no SISCEL e SINAN e 45 no SISCEL e SIM (Figura 1).

Na base de dados de crianças notificadas com HIV/AIDS, o percentual de municípios "silenciosos”, ou seja, aqueles que não apresentaram nenhuma notificação de HIV/AIDS em crianças 
Tabela 1

Características sociodemográficas, clínicas e de tratamento das gestantes notificadas por HIV na gestação em cinco estados e no Distrito Federal, Brasil, 2007-2012.

\begin{tabular}{|c|c|c|c|c|c|c|c|}
\hline Características & $\begin{array}{c}\text { AM } \\
{[n=1.255]}\end{array}$ & $\begin{array}{c}\text { CE } \\
{[n=1.082]}\end{array}$ & $\begin{array}{c}\text { DF } \\
{[n=308]}\end{array}$ & $\begin{array}{c}\text { ES } \\
{[n=636]}\end{array}$ & $\begin{array}{c}\text { RJ } \\
{[n=3.903]}\end{array}$ & $\begin{array}{c}\text { RS } \\
{[n=6.870]}\end{array}$ & Valor de $p$ \\
\hline Idade em anos (mediana, IQR) & $23(20-29)$ & $26(22-30)$ & $27(23-32)$ & $27(22-31)$ & $26(22-31)$ & $27(23-32)$ & 0,002 \\
\hline \multicolumn{8}{|l|}{ Escolaridade (anos) [n (\%)] } \\
\hline Até 8 & $611(48,7)$ & $597(55,2)$ & $150(48,7)$ & $305(48,0)$ & $1.898(48,6)$ & $4.585(66,7)$ & $<0,001$ \\
\hline 9 e mais & $393(31,3)$ & $290(26,8)$ & $99(32,1)$ & $172(27,0)$ & $847(21,7)$ & $1.001(14,6)$ & \\
\hline Ignorado & $251(20,0)$ & $195(18,0)$ & $59(19,2)$ & $159(25,0)$ & $1.158(29,7)$ & $1.284(18,7)$ & \\
\hline \multicolumn{8}{|l|}{ Raça/Cor [n (\%)] } \\
\hline Branca & $142(11,3)$ & $163(15,1)$ & $84(27,3)$ & $132(20,8)$ & $1.042(26,7)$ & $4.425(64,4)$ & $<0,001$ \\
\hline Preta & $102(8,1)$ & $77(7,1)$ & $45(14,6)$ & $114(17,9)$ & $992(25,4)$ & $1.380(20,1)$ & \\
\hline Amarela & $18(1,4)$ & $16(1,5)$ & $1(0,3)$ & $8(1,3)$ & $32(0,8)$ & $23(0,3)$ & \\
\hline Parda & $936(74,6)$ & $763(70,5)$ & $156(50,7)$ & $360(56,5)$ & $1.340(34,3)$ & $760(11,1)$ & \\
\hline Indígena & $16(1,3)$ & $1(0,1)$ & $1(0,3)$ & $0(0,0)$ & $10(0,3)$ & $15(0,2)$ & \\
\hline Ignorado & $41(3,3)$ & $62(5,7)$ & $21(6,8)$ & $22(3,5)$ & $487(12,5)$ & $267(3,9)$ & \\
\hline \multicolumn{8}{|l|}{ Local de residência [n (\%)] } \\
\hline Capital & $1.048(83,5)$ & $569(52,6)$ & $308(100,0)$ & $81(12,7)$ & $2.082(53,3)$ & $2.271(33,1)$ & $<0,001$ \\
\hline Interior & $207(16,5)$ & $513(47,4)$ & $0(0,0)$ & $555(87,3)$ & $1.821(46,7)$ & $4.599(66,9)$ & \\
\hline \multicolumn{8}{|l|}{ Resultado HIV [n (\%)] } \\
\hline Antes do pré-natal & $278(22,1)$ & $405(37,4)$ & $151(49,0)$ & $365(57,4)$ & $1.488(38,1)$ & $3.640(53,0)$ & $<0,001$ \\
\hline No pré-natal & $671(53,5)$ & $556(51,4)$ & $131(42,5)$ & $213(33,5)$ & $1.783(45,7)$ & $2.553(37,2)$ & \\
\hline No parto & $246(19,6)$ & $102(9,4)$ & $20(6,5)$ & $47(7,4)$ & $467(12,0)$ & $591(8,6)$ & \\
\hline Pós-parto & $60(4,8)$ & $19(1,8)$ & $6(2,0)$ & $11(1,7)$ & $165(4,2)$ & $86(1,2)$ & \\
\hline \multicolumn{8}{|l|}{ Realizou pré-natal [n (\%)] } \\
\hline Sim & $991(79,0)$ & $972(89,8)$ & $279(90,6)$ & $579(91,0)$ & $3.288(84,3)$ & $6.026(87,7)$ & $<0,001$ \\
\hline Não & $203(16,2)$ & $72(6,6)$ & $26(8,4)$ & $43(6,8)$ & $391(10,0)$ & $702(10,2)$ & \\
\hline Ignorado & $61(4,8)$ & $38(3,5)$ & $3(1,0)$ & $14(2,2)$ & $224(5,7)$ & $142(2,1)$ & \\
\hline \multicolumn{8}{|l|}{ Uso TARV pré-natal [n (\%)] } \\
\hline Sim & $522(41,6)$ & $713(65,9)$ & $236(76,6)$ & $491(77,2)$ & $2.301(59,0)$ & $4.672(68,0)$ & $<0,001$ \\
\hline Não & $237(18,9)$ & $188(17,4)$ & $28(9,1)$ & $61(9,6)$ & $563(14,4)$ & $1.010(14,7)$ & \\
\hline Ignorado & $496(39,5)$ & $181(16,7)$ & $44(14,3)$ & $84(13,2)$ & $1.039(26,6)$ & $1.188(17,3)$ & \\
\hline \multicolumn{8}{|l|}{ Uso TARV durante oparto [n (\%)] } \\
\hline Sim & $475(37,9)$ & $739(68,3)$ & $217(70,4)$ & $376(59,1)$ & $1.989(51,0)$ & $5.097(74,2)$ & $<0,001$ \\
\hline Não & $216(17,2)$ & $113(10,4)$ & $48(15,6)$ & $80(12,6)$ & $572(14,7)$ & $790(11,5)$ & \\
\hline Ignorado & $564(44,9)$ & $230(21,3)$ & $43(14,0)$ & $180(28,3)$ & $1.342(34,3)$ & $983(14,3)$ & \\
\hline \multicolumn{8}{|l|}{ Tipo de parto [n (\%)] } \\
\hline Vaginal & $168(13,4)$ & $143(13,2)$ & $35(11,4)$ & $97(15,2)$ & $882(22,6)$ & $2.202(32,0)$ & $<0,001$ \\
\hline Cesárea eletiva & $406(32,3)$ & $622(57,5)$ & $189(61,4)$ & $270(42,5)$ & $1.401(35,9)$ & $3.240(47,2)$ & \\
\hline Cesárea urgência & $54(4,3)$ & $73(6,8)$ & $25(8,1)$ & $71(11,2)$ & $261(6,7)$ & $294(4,3)$ & \\
\hline Não se aplica/lgnorado & $627(50,0)$ & $244(22,5)$ & $59(19,1)$ & $198(31,1)$ & $1.358(34,8)$ & $1.134(16,5)$ & \\
\hline \multicolumn{8}{|l|}{ Produto do parto [n (\%)] } \\
\hline Nascido vivo & $624(49,7)$ & $833(77,0)$ & $248(80,5)$ & $437(68,7)$ & $2.546(65,2)$ & $5.736(83,5)$ & \\
\hline Natimorto & $16(1,3)$ & $20(1,9)$ & $6(2,0)$ & $12(1,9)$ & $45(1,2)$ & $125(1,8)$ & \\
\hline Aborto & $46(3,7)$ & $9(0,8)$ & $9(2,9)$ & $11(1,7)$ & $109(2,8)$ & $173(2,5)$ & \\
\hline Ignorado & $569(45,3)$ & $220(20,3)$ & $45(14,6)$ & $176(27,7)$ & $1.203(30,8)$ & $836(12,2)$ & \\
\hline
\end{tabular}

(continua) 
Tabela 1 (continuação)

\begin{tabular}{|c|c|c|c|c|c|c|c|}
\hline Características & $\begin{array}{c}\text { AM } \\
{[n=1.255]}\end{array}$ & $\begin{array}{c}\text { CE } \\
{[n=1.082]}\end{array}$ & $\begin{array}{c}\text { DF } \\
{[n=308]}\end{array}$ & $\begin{array}{c}\text { ES } \\
{[n=636]}\end{array}$ & $\begin{array}{c}\text { RJ } \\
{[n=3.903]}\end{array}$ & $\begin{array}{c}\text { RS } \\
{[n=6.870]}\end{array}$ & Valor de $p$ \\
\hline \multicolumn{8}{|c|}{ Uso de TARV pelo recém nascido [n (\%)] } \\
\hline Nas primeiras 24 horas & $564(90,4)$ & $788(94,6)$ & $218(87,9)$ & $412(94,3)$ & $2.306(90,6)$ & $5.489(95,7)$ & \\
\hline$>24$ horas & $18(2,9)$ & $16(1,9)$ & $2(0,8)$ & $4(0,9)$ & $45(1,7)$ & $34(0,6)$ & \\
\hline Não se aplica & $2(0,3)$ & $1(0,1)$ & $0(0,0)$ & $0(0,0)$ & $3(0,1)$ & $7(0,1)$ & \\
\hline Não realizado & $10(1,6)$ & $10(1,2)$ & $4(1,6)$ & $3(0,7)$ & $10(0,4)$ & $28(0,5)$ & \\
\hline Ignorado & $30(4,8)$ & $18(2,2)$ & $24(9,7)$ & $18(4,1)$ & $182(7,2)$ & $178(3,1)$ & \\
\hline
\end{tabular}

AM: Amazonas; CE: Ceará; DF: Distrito Federal; ES: Espírito Santo; IQR: interquartile range; RJ: Rio de Janeiro;

RS: Rio Grande do Sul; TARV: terapia antirretroviral.

Fonte: Ministério da Saúde. Sistema de Informação de Agravos de Notificação.

Tabela 2

Distribuição proporcional e intervalo de 95\% de confiança (IC95\%) da cascata de cuidado para a profilaxia da transmissão vertical do HIV por momento de diagnóstico do HIV materno e por Unidade de Federação, Brasil, 2007-2012.

\begin{tabular}{|c|c|c|c|c|c|c|}
\hline Cascata de cuidado & $\begin{array}{c}\text { AM } \\
{[n=278]}\end{array}$ & $\begin{array}{c}\text { CE } \\
{[n=405]}\end{array}$ & $\begin{array}{c}\text { DF } \\
{[n=151]}\end{array}$ & $\begin{array}{c}\text { ES } \\
{[n=365]}\end{array}$ & $\begin{array}{c}\text { RJ } \\
{[n=1,488]}\end{array}$ & $\begin{array}{c}\text { RS } \\
{[n=3,640]}\end{array}$ \\
\hline \multicolumn{7}{|l|}{ Diagnóstico de HIV antes do pré-natal } \\
\hline Realizou pré-natal & $\begin{array}{c}81,7 \\
(77,1-86,2)\end{array}$ & $\begin{array}{c}88,6 \\
(85,5-91,7)\end{array}$ & $\begin{array}{c}92,1 \\
(87,7-96,4)\end{array}$ & $\begin{array}{c}94,2 \\
(91,8-96,6)\end{array}$ & $\begin{array}{c}88,4 \\
(86,7-90,0)\end{array}$ & $\begin{array}{c}89,0 \\
(88,0-90,1)\end{array}$ \\
\hline TARV durante o pré-natal & $\begin{array}{c}68,3 \\
(62,2-74,4)\end{array}$ & $\begin{array}{c}83,6 \\
(79,7-87,4)\end{array}$ & $\begin{array}{c}89,9 \\
(84,9-95,0)\end{array}$ & $\begin{array}{c}89,8 \\
(86,6-93,0)\end{array}$ & $\begin{array}{c}82,6 \\
(80,5-84,6)\end{array}$ & $\begin{array}{c}84,5 \\
(83,3-85,8)\end{array}$ \\
\hline TARV durante o parto & $\begin{array}{c}43,2 \\
(35,3-51,1)\end{array}$ & $\begin{array}{c}82,3 \\
(78,0-86,7)\end{array}$ & $\begin{array}{c}78,4 \\
(71,1-85,7)\end{array}$ & $\begin{array}{c}62,1 \\
(56,7-67,6)\end{array}$ & $\begin{array}{c}63,3 \\
(60,4-66,1)\end{array}$ & $\begin{array}{c}80,2 \\
(78,7-81,7)\end{array}$ \\
\hline Cesárea eletiva & $\begin{array}{c}77,6 \\
(67,4-87,9)\end{array}$ & $\begin{array}{c}87,9 \\
(83,8-92,0)\end{array}$ & $\begin{array}{c}81,6 \\
(73,8-89,4)\end{array}$ & $\begin{array}{c}68,8 \\
(62,1-75,4)\end{array}$ & $\begin{array}{c}61,7 \\
(58,1-65,4)\end{array}$ & $\begin{array}{c}60,2 \\
(58,1-62,2)\end{array}$ \\
\hline TARV pelo recém-nascido nas primeiras 24 horas de vida & $\begin{array}{c}96,2 \\
(90,7-100,0)\end{array}$ & $\begin{array}{c}96,8 \\
(94,4-99,1)\end{array}$ & $\begin{array}{c}90,0 \\
(83,3-96,7)\end{array}$ & $\begin{array}{c}99,2 \\
(97,7-100,0)\end{array}$ & $\begin{array}{c}97,4 \\
(95,9-98,9)\end{array}$ & $\begin{array}{c}98,0 \\
(97,3-98,8)\end{array}$ \\
\hline Diagnóstico de HIV no pré-natal & {$[n=671]$} & {$[n=556]$} & {$[n=131]$} & {$[n=213]$} & {$[n=1.783]$} & {$[n=2.553]$} \\
\hline TARV durante o pré-natal & $\begin{array}{c}55,7 \\
(51,8-59,7)\end{array}$ & $\begin{array}{c}75,4 \\
(71,7-79,0)\end{array}$ & $\begin{array}{c}86,4 \\
(80,3-92,5)\end{array}$ & $\begin{array}{c}87,6 \\
(83,0-92,2)\end{array}$ & $\begin{array}{c}70,5 \\
(68,3-72,7)\end{array}$ & $\begin{array}{c}77,2 \\
(75,5-78,8)\end{array}$ \\
\hline TARV durante o parto & $\begin{array}{c}41,1 \\
(35,8-46,3)\end{array}$ & $\begin{array}{c}72,6 \\
(68,2-77,0)\end{array}$ & $\begin{array}{c}75,0 \\
(66,7-83,3)\end{array}$ & $\begin{array}{c}68,8 \\
(61,8-75,7)\end{array}$ & $\begin{array}{c}60,0 \\
(57,0-62,5)\end{array}$ & $\begin{array}{c}76,8 \\
(74,9-78,7)\end{array}$ \\
\hline Cesárea eletiva & $\begin{array}{c}80,0 \\
(73,3-86,7)\end{array}$ & $\begin{array}{c}83,5 \\
(79,2-87,8)\end{array}$ & $\begin{array}{c}87,7 \\
(80,3-95,0)\end{array}$ & $\begin{array}{c}76,0 \\
(68,3-83,7)\end{array}$ & $\begin{array}{c}66,9 \\
(63,4-70,4)\end{array}$ & $\begin{array}{c}62,5 \\
(60,0-65,0)\end{array}$ \\
\hline TARV pelo recém-nascido nas primeiras 24 horas de vida & $\begin{array}{c}99,1 \\
(97,3-100,0)\end{array}$ & $\begin{array}{c}97,5 \\
(95,6-99,5)\end{array}$ & $\begin{array}{c}93,0 \\
(86,9-99,1)\end{array}$ & $\begin{array}{c}91,3 \\
(85,4-97,2)\end{array}$ & $\begin{array}{c}96,4 \\
(94,7-98,1)\end{array}$ & $\begin{array}{c}97,7 \\
(96,7-98,7)\end{array}$ \\
\hline Diagnóstico de HIV no parto & {$[n=246]$} & {$[n=102]$} & {$[n=20]$} & {$[n=47]$} & {$[n=467]$} & {$[n=591]$} \\
\hline Realizou pré-natal & $\begin{array}{c}51,6 \\
(45,3-57,9)\end{array}$ & $\begin{array}{c}65,7 \\
(56,3-75,1)\end{array}$ & $\begin{array}{c}55,0 \\
(31,1-78,9)\end{array}$ & $\begin{array}{c}59,6 \\
(45,0-74,1)\end{array}$ & $\begin{array}{c}45,8 \\
(41,2-50,4)\end{array}$ & $\begin{array}{c}55,3 \\
(51,3-59,3)\end{array}$ \\
\hline TARV durante o parto & $\begin{array}{c}60,2 \\
(54,0-66,3)\end{array}$ & $\begin{array}{c}75,5 \\
(67,0-84,0)\end{array}$ & $\begin{array}{c}60,0 \\
(36,5-83,5)\end{array}$ & $\begin{array}{c}59,6 \\
(45,0-74,1)\end{array}$ & $\begin{array}{c}46,9 \\
(42,4-51,4)\end{array}$ & $\begin{array}{c}71,7 \\
(68,1-75,4)\end{array}$ \\
\hline TARV pelo recém-nascido nas primeiras 24 horas de vida & $\begin{array}{c}98,6 \\
(96,7-100,0)\end{array}$ & $\begin{array}{c}96,0 \\
(91,4-100,0)\end{array}$ & 100,0 & $\begin{array}{c}96,4 \\
(89,1-100,0)\end{array}$ & $\begin{array}{c}94,4 \\
(91,3-97,5)\end{array}$ & $\begin{array}{c}97,2 \\
(95,6-98,7)\end{array}$ \\
\hline
\end{tabular}

AM: Amazonas; CE: Ceará; DF: Distrito Federal; ES: Espírito Santo; RJ: Rio de Janeiro; RS: Rio Grande do Sul; TARV: terapia antirretroviral.

Fonte: Ministério da Saúde. Sistema de Informação de Agravos de Notificação. 


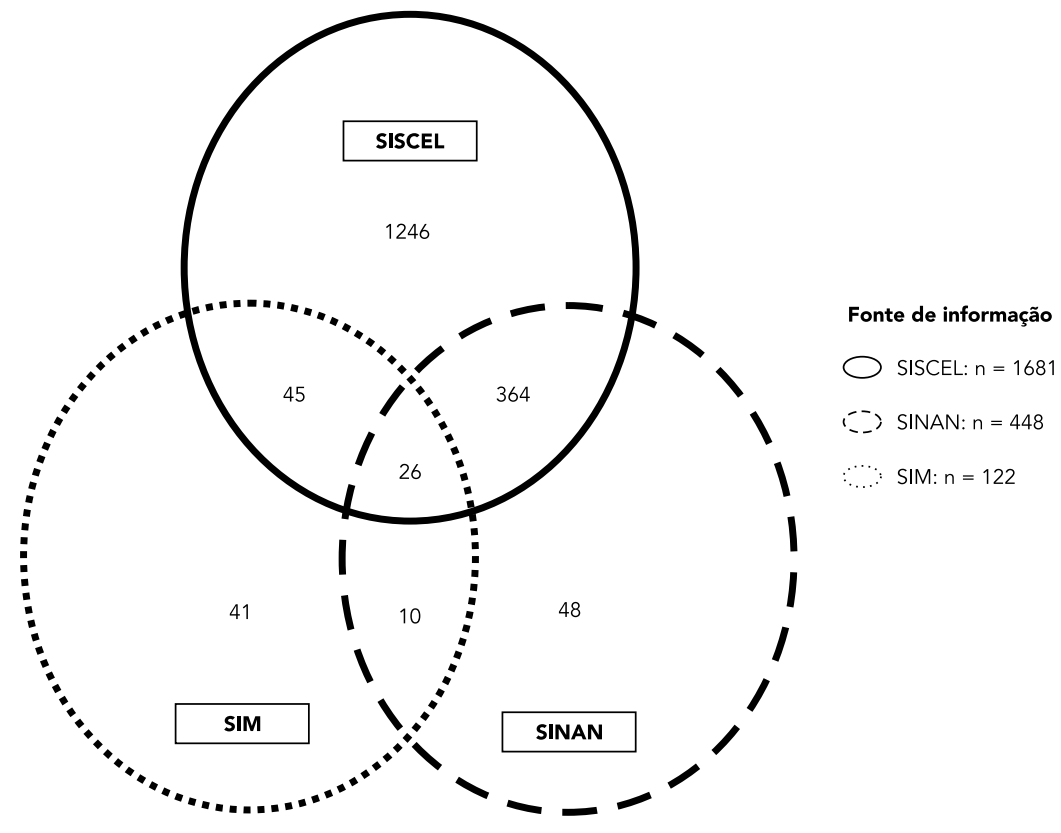

SIM: Sistema de Informação sobre Mortalidade; SINAN: Sistema de Informação de Agravos de Notificação; SISCEL: Sistema de Controle de Exames Laboratoriais.

no período foi de $69,4 \%$ no Amazonas, $77,7 \%$ no Ceará, $59 \%$ no Espírito Santo, 48,9\% no Rio de Janeiro e 78,3\% no Rio Grande do Sul.

Dos 185 casos de HIV/AIDS em crianças no Amazonas, 84,3\% residiam na capital, assim como $52 \%$ dos 127 casos do Ceará, 11,2\% dos 161 do Espírito Santo, 46,3\% dos 529 do Rio de Janeiro e 31,8\% dos 746 do Rio Grande do Sul. O Distrito Federal contribuiu com 22 casos.

A taxa de detecção de AIDS em crianças menores de 5 anos reduziu-se em 6,3\% entre 2007 e 2012. A maior redução se deu no Rio Grande do Sul (50\%), que apresentou as maiores taxas do período, enquanto o maior aumento ocorreu no Espírito Santo, de 50\% (Figura 2).

\section{Discussão}

Desde que a utilização dos antirretrovirais se mostrou efetiva em reduzir a transmissão vertical do HIV 7, algumas intervenções passaram a ser recomendadas para serem iniciadas no acesso da gestante ao pré-natal. O presente estudo aponta alta cobertura de pré-natal entre as gestantes in- fectadas pelo HIV (variando de 79 a 91\%), abaixo da relatada por Domingues et al. 10, com quase 99\% com pelo menos uma consulta. Esses autores encontraram $81,7 \%$ de realização de teste anti-HIV no pré-natal, com uma prevalência de $0,4 \%$ de HIV nas gestantes.

Entre os estudos brasileiros, encontra-se um paradoxo. Há estudos de base hospitalar (ou de centros de referência) e estudos utilizando dados secundários com resultados divergentes, por conta da seleção prévia de gestantes já com diagnóstico de HIV nos primeiros, e a adição de casos notificados sem nenhuma intervenção para redução da transmissão vertical do HIV nos dados secundários. Exemplos de sucesso no Rio de Janeiro, com 2,8\% de transmissão em uma coorte com 145 crianças 15 e outra com 3,6\% entre 297 crianças 16; em Belo Horizonte, 939 crianças resultaram em 3\% 17; e 4,9\% de infeção entre 144 crianças em Rio Grande do Sul 18. Já em Pernambuco, 1.200 crianças expostas foram seguidas entre 2000 e 2009, resultando em 9,2\% infectadas pelo HIV 19, comparável aos achados do Amazonas entre 2007 e 2009 14, com dados do SINAN. Porém, em 1998, a taxa de transmissão vertical 


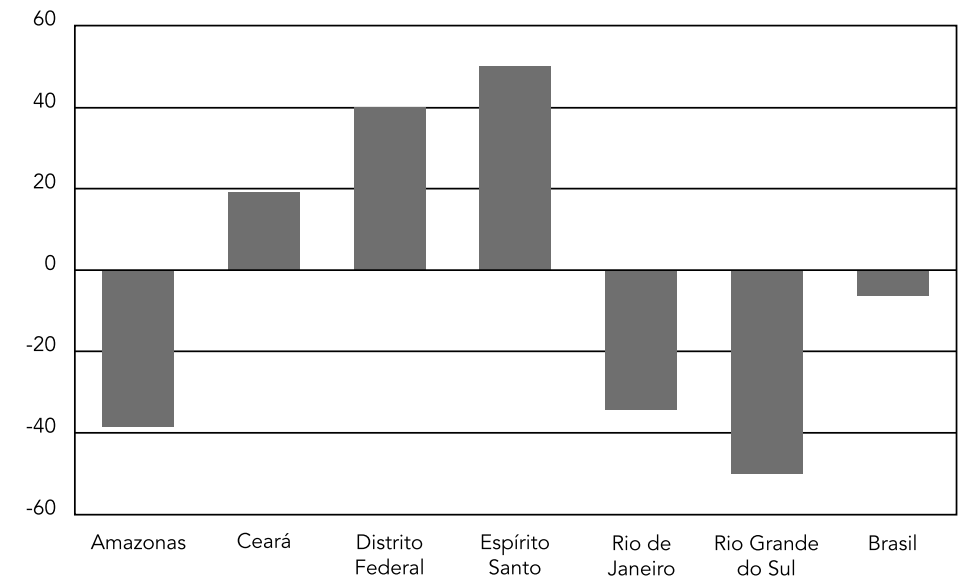

no Estado de São Paulo foi de $16 \%$ em estudo de base hospitalar 20. Em 2006, no mesmo estado, houve uma redução para $2,7 \%$, utilizando-se dados secundários, o que pode ser considerado um grande sucesso programático 21 .

Essas diferenças refletem as falhas na cascata de cuidado para a prevenção da transmissão vertical HIV, que neste estudo estiveram presente, não importando a época do diagnóstico materno de HIV. Quase 20\% das mulheres no Amazonas chegaram ao parto sem saber seu status HIV, concordando com outro estudo nacional, em que $29 \%$ das mulheres não foram testadas durante o pré-natal ${ }^{12}$. As regiões Norte e Nordeste apresentaram as menores prevalências de realização do primeiro teste anti-HIV no pré-natal, respectivamente, $69,9 \%$ e $68,4 \%$, apontando para uma menor adesão aos protocolos de redução de transmissão vertical do HIV 10. Em nenhuma das seis Unidades de Federação foi possível identificar o uso de TARV durante o pré-natal em proporção superior a $90 \%$ das mulheres notificadas, mesmo entre aquelas que já se sabiam portadoras do HIV. Foi ainda mais difícil conseguir realizar o parto como cesárea eletiva previamente agendada, e o uso da TARV pelo recém-nascido nas primeiras 24 horas também ficou muito aquém do desejado, quando avaliado pela cascata completa de cuidado. Na região metropolitana do Rio de Janeiro, apenas 52,7\% das mulheres atendidas em um hospital de referência receberam todo o pacote de intervenções necessárias para a prevenção da transmissão vertical HIV 13 .
Em São Paulo, em hospital universitário, falhas nos fluxos de referência e de atendimento foram identificadas, sendo menores para o HIV do que para a sífilis 22 .

A utilização de testes rápidos no parto e no pré-natal 23 deveria ser uma estratégia para corrigir falhas programáticas e recolocar mulheres infectadas com HIV em um curso favorável para um bom desfecho da gestação. A rede de serviços destinados a prestar assistência às mulheres com HIV/AIDS deve estar articulada às unidades de atenção básica, local onde deve se iniciar todo processo de cuidado, considerando que se trata do espaço no qual é feito o diagnóstico de gravidez e consequentemente a solicitação dos exames de rotina, dentre eles a testagem para o HIV 5 .

Como pode ser constatado, a cascata de cuidados para a redução da transmissão vertical do HIV apresentou perdas de seguimento relevantes, que podem estar relacionadas a questões não listadas no modelo biomédico dessa cascata. Uma revisão sistemática das ações para a redução da transmissão vertical do HIV na China entre 2003 e 2011 mostrou uma queda da transmissão vertical de $12,9 \%$ para $2,3 \%$ no período. Contudo, ao incluir as mulheres que não foram captadas pela cascata de cuidado, esses valores subiram para $27,1 \%$ e $11,5 \%$, respectivamente 24 . Um modelo que serve a todos não tem se mostrado eficiente no cuidado dispensado aos portadores de HIV. Um enfoque centrado nas trajetórias individuais dos pacientes, focando nas vulnerabilidades 
de cada um, pode reduzir as perdas 25 . Pelo visto neste estudo, o Brasil poderia se beneficiar desse enfoque em trajetórias, pois conta tanto com um ativismo comunitário em HIV/AIDS com grande experiência, como também com a expansão da Estratégia Saúde da Família, que congrega a atuação de agentes comunitários de saúde com um olhar das equipes sobre as famílias, o que reduziria as perdas ao atender melhor o indivíduo em seu cenário de vida.

O fato de o SINAN não ter condições de armazenar a ficha de investigação de criança exposta traz mais uma barreira programática ao conjunto de barreiras a serem superadas. A informação proveniente da notificação de casos de AIDS em crianças menores de 13 anos é demorada, e só recentemente a notificação de HIV, além da de AIDS, foi introduzida no Brasil 1. O uso de múltiplas fontes para resgatar a informação de casos de AIDS contribui muito, mas não substitui o quadro mais acurado que se teria caso o seguimento de todas as crianças expostas estivesse disponível. Portanto, lidar com um proxy, o indicador de taxa de detecção de AIDS em menores de 5 anos ainda pode estar subestimando a real taxa de transmissão vertical HIV, mascarando ainda mais um quadro que já se desenha, de uma população mais vulnerável, que não alcança e não é alcançada pelos serviços de saúde.

\section{Colaboradores}

A. E. Miranda contribuiu na concepção e delineamento do estudo, revisão da análise, escrita do artigo, aprovação da forma final. G. F. M. Pereira colaborou na revisão de dados, revisão final do artigo. M. A. L. Araujo participou na concepção do estudo, revisão crítica do artigo. M. F. Silveira contribuiu na concepção e delineamento do estudo, aprovação da forma final do artigo. L. L. Tavares, L. C. F. Silva e S. F. Moreira-Silva colaboraram na coleta de dados, revisão crítica do artigo. V. Saraceni contribuiu na concepção e delineamento do estudo, coleta e análise de dados, revisão crítica, aprovação final do artigo.
O percentual de respostas ignoradas para variáveis relacionadas ao uso de TARV nas várias etapas, especialmente nos estados do Amazonas e Rio de Janerio, pode ter reduzido a capacidade de avaliação do conjunto. Todavia, a possibilidade de avaliar as ações propostas para a prevenção da transmissão vertical do HIV em base populacional se justifica ao se notar que ainda não se atingiu a redução desejada na transmissão vertical, embora as estratégias estejam traçadas e os protocolos disponíveis.

\section{Conclusões}

Os dados secundários ainda apontam falhas importantes na cascata de cuidados para prevenção da transmissão vertical do HIV, mostrando que oportunidades são perdidas nos diferentes serviços e em todas as etapas da assistência à mulher, sobretudo após a descoberta do diagnóstico de HIV. Uma conexão forte entre a atenção básica e os centros de referência para HIV/AIDS se faz necessária, tendo em conta a importância da primeira como ordenadora do cuidado da família sob sua responsabilidade. A melhoria da completude dos dados de notificação permitirá uma melhor avaliação de uma política pública que sabidamente resulta em grande impacto na saúde da criança.

\section{Agradecimentos}

Ao Ministério da Saúde, Secretaria Executiva, Fundação Nacional de Saúde Termo de Cooperação no 323/201. 


\section{Referências}

1. Programa Nacional de DST e Aids, Secretaria de Vigilância em Saúde, Ministério da Saúde. Boletim Epidemiológico Aids e DST. Brasília: Ministério da Saúde; 2014.

2. Brito AM, Castilho EA, Szwarcwald CL. AIDS e infecção pelo HIV no Brasil: uma epidemia multifacetada. Rev Soc Bras Med Trop 2001; 34:207-17.

3. Fonseca MGP, Bastos FI. Twenty-five years of the AIDS epidemic in Brazil: principal epidemiological findings, 1980-2005. Cad Saúde Pública 2007; 23 Suppl 3:S333-44.

4. Fonseca MGP, Szwarcwald CL, Bastos FI. Análise sociodemográfica de AIDS no Brasil, 1989-1997. Rev Saúde Pública 2002; 36:678-85.

5. Programa Nacional de DST e Aids, Secretaria de Vigilância em Saúde, Ministério da Saúde. Recomendações para profilaxia da transmissão vertical do HIV e terapia antirretroviral em gestantes. Brasília: Ministério da Saúde; 2010.

6. Joint United Nations Program on HIV/AIDS. AIDSinfo. Banco de dados sobre AIDS. A epidemia de AIDS no mundo. http://www.unaids.org.br/sobre aids/sobre_aids.asp (acessado em 15/Jul/2015).

7. Connor EM, Sperling RS, Gelber R, Kiselev P, Scott G, O'Sullivan MJ et al. Reduction of maternal-infant transmission of human immunodeficiency virus type 1 with zidovudine treatment. Pediatric AIDS Clinical Trials Group Protocol 076 Study Group. N Engl J Med 1994; 331:1173-80.

8. Joint United Nations Program on HIV/AIDS. Progress report on the global plan. http://www.unaids. org/en/media/unaids/contentassets/documents/ unaidspublication/2013/20131129_stocktaking_ report_children_aids_en.pdf (acessado em 11/ Jan/2016)

9. Souza-Júnior PRB, Szwarcwald CL, BarbosaJunior A, Carvalho MF, Castilho EA. Infecção pelo HIV durante a gestação: Estudo-Sentinela Parturiente, Brasil, 2002. Rev Saúde Pública 2004; 38:764-772.

10. Domingues RM, Szwarcwald CL, Souza PR Jr, Leal Mdo C. Prenatal testing and prevalence of HIV infection during pregnancy: data from the "Birth in Brazil" study, a national hospital-based study. BMC Infect Dis 2015; 15:100.

11. Gardner EM, McLees MP, Steiner JF, del Rio C, Burman WJ. The spectrum of engagement in HIV care and its relevance to test and-treat strategies for prevention of HIV infection. Clin Infect Dis 2011; 52:793-800.

12. Rodrigues CS, Guimarães MD, César CC. Missed opportunities for congenital syphilis and HIV perinatal transmission prevention. Rev Saúde Pública 2008; 42:851-8.

13. Araujo ESP, Friedman RK, Camacho LAB, Derrico M, Moreira RI, Calvet GA, et al. Cascade of access to interventions to prevent HIV mother to child transmission in the metropolitan area of Rio de Janeiro, Brazil. Braz J Infect Dis 2014; 18:252-60.

14. Soeiro CM, Miranda AE, Saraceni V, Lucena NO Talhari S, Ferreira LC. Mother-to-child transmission of HIV infection in Manaus, State of Amazonas, Brazil. Rev Soc Bras Med Trop 2011; 44:537-41.
15. Nogueira SA, Abreu T, Oliveira R, Araújo L, Costa T, Andrade M, et al. Successful prevention of HIV transmission from mother to infant in Brazil using a multidisciplinary team approach. Braz J Infect Dis 2001; 5:78-86.

16. João EC, Cruz ML, Menezes JA, Matos HJ, Calvet GA, d'Ippolito MM, et al. Vertical transmission of HIV in Rio de Janeiro, Brazil. AIDS 2003; 17:1853-5.

17. Kakehasi FM, Pinto JA, Romanelli RM, Carneiro M, Cardoso CS, Tavares MC, et al. Determinants and trends in perinatal human immunodeficiency virus type 1 (HIV-1) transmission in the metropolitan area of Belo Horizonte, Brazil: 1998 - 2005. Mem Inst Oswaldo Cruz 2008; 103:351-7.

18. Tornatore M, Gonçalves CV, Mendoza-Sassi RA, Silveira JM, D'ávila NE, Maas CG, et al. HIV-1 vertical transmission in Rio Grande, Southern Brazil. Int J STD AIDS 2010; 21:351-5.

19. Gouveia PAC, Silva GA, Albuquerque MFPM. Factors associated with mother-to-child transmission of the human immunodeficiency virus in Pernambuco, Brazil, 2000-2009. Trop Med Int Health 2013; 18:276-85.

20. Tess BH, Rodrigues LC, Newell ML, Dunn DT, Lago TD. Breastfeeding, genetic, obstetric and other risk factors associated with mother-to-child transmission of HIV-1 in Sao Paulo State, Brazil. Sao Paulo Collaborative Study for Vertical Transmission of HIV-1. AIDS 199; 12:513-20.

21. Matida LH, Santos NJ, Ramos AN Jr, Gianna MC, da Silva MH, Domingues CS, et al. Eliminating vertical transmission of HIV in São Paulo, Brazil: progress and challenges. J Acquir Immune Defic Syndr 2011; 57 Suppl 3:S164-70.

22. Ramos VM, Figueiredo EN, Succi RC. Barriers to control syphilis and HIV vertical transmission in the health care system in the city of Sao Paulo. Rev Bras Epidemiol 2014; 17:887-98.

23. Veloso VG, Bastos FI, Portela MC, Grinsztejn B, João EC, Pilotto JH, et al. HIV rapid testing as a key strategy for prevention of mother-to-child transmission in Brazil. Rev Saúde Pública 2010; 44:803-11.

24. Zeng H, Chow EP, Zhao Y, Wang Y, Tang M, Li L, et al. Prevention of mother-to-child HIV transmission cascade in China: a systematic review and meta-analysis. Sex Transm Infect 2016; 92:116-23.

25. Hsieh A, Rodrigues J, Skovdal M, Melillo S, Walker D; Community Engagement Working Group of the Interagency Task Team on the Prevention and Treatment of HIV Infection in Pregnant Women, Mothers and Children. From patient to person: the need for an 'HIV trajectories' perspective in the delivery of prevention of mother-to-child-transmission services. AIDS 2014; 28 Suppl 3:S399-409. 


\section{Abstract}

This study aimed to assess the cascade of care in the reduction of mother-to-child HIV transmission in the states of Amazonas, Ceará, Espírito Santo, Rio de Janeiro, and Rio Grande do Sul and the Distrito Federal, Brazil, using data from the Brazilian Information System on Diseases of Notification (SINAN). From 2007 to 2012, there was an increase (from $7.3 \%$ in Distrito Federal to $46.1 \%$ in Amazonas) in intra-gestational detection of HIV in 5 states, with a $18.6 \%$ reduction in Rio de Janeiro. Fewer than $90 \%$ of the women received antiretroviral therapy during their prenatal care, including those that already knew they were HIV-positive. The elective cesarean rate was low. The AIDS detection rate in children under 5 years as a proxy for mother-to-child HIV transmission showed a reduction of 6.3\% from 2007 to 2012, and was highest in Rio Grande do Sul (50\%), the state with the highest rates in the period, while Espirito Santo showed the highest increase (50\%). Evaluation of the cascade of HIV care in pregnant women identified flaws in all the points. A link is needed between primary care and referral centers for HIVIAIDS, organizing care for the family and better outcomes for the children.

HIV; Pregnancy; Infectious Disease Vertical Transmission; Primary Health Care

\section{Resumen}

Este estudio tuvo por objetivo evaluar la cascada de cuidado de la reducción de la transmisión vertical del VIH en los Estados del Amazonas, Ceará, Espírito Santo, Río de Janeiro, Río Grande do Sul y en el Distrito Federal, usando datos del Sistema de Información de Enfermedades de Notificación Obligatoria (SINAN por sus siglas en portugués). Entre los años de 2007 y 2012 creció la tasa de detección de VIH durante la gestación en 5 estados, variando de 7,3\% en el Distrito Federal a 46,1\% en Amazonas, con una reducción de 18,6\% en Río de Janeiro. Menos de un 90\% de las mujeres usaron antirretrovirales durante el período prenatal, incluidas quienes ya se sabían portadoras del VIH. La realización de cesárea electiva fue baja. La tasa de detección de SIDA en niños menores de 5 años como proxy de la transmisión vertical del VIH presentó una reducción de 6,3\% entre 2007 y 2012, siendo la mayor en Río Grande do Sul (50\%), la cual presentó las mayores tasas del período, mientras que en Espírito Santo se produjo el mayor aumento (50\%). La evaluación de la cascada del cuidado del VIH en la gestante apuntó fallos en todos los puntos. Es necesaria una conexión entre la atención básica y los centros de referencia para VIH/SIDA, buscando el cuidado de la familia y el mejor desenlace para el niños.

VIH: Embarazo; Transmisión Vertical de Enfermedad Infecciosa; Atención Primaria de Salud
Recebido em 21/Jul/2015

Versão final reapresentada em 19/Jan/2016 Aprovado em 24/Fev/2016 